\title{
Land Evaluation of a New Reclaimed Area, South of El-Tina Plain, Egypt using a Proposed System and GIS
}

\author{
M. A. Eid, Y.M. Zakarya and M. S. Abd-Elwahed \\ * Soil Science Department, Faculty, Agriculture, Ain Shams \\ University, Cairo, Egypt.
}

\begin{abstract}
T HE MAIN goal of this study is to assess the land spatial variability and evaluate soils of $127.29 \mathrm{Km}^{2}$ for irrigated agriculture in the new reclamation area, El-Tina Plain, Egypt. The detailed objectives of this research are: to assess the land spatial variability using GIS modeling, to evaluate the main soil units using proposed system integrated with GIS and to identify the main limitation factors in the study area. Field survey was carried out to characterize each land unit. Eighteen soil profiles were dug in the field, morphologically described and sampled for laboratory analyses. The soils in the study area were classified as; Typic Aquisalids, Typic Torriorthents and Typic Torripsamments. The capability process was done using a modified local system and GIS modeling. The utilized evaluation system was developed based on the mathematical modeling of different land evaluation parameters (soil physical, chemical and irrigation water quality). Interpolation of different soil characteristics was done to create different soil maps. The final capability map was created through the overlaying process using the interpolated maps. The study area was classified as $80.6 \%$ belongs to S3 (Marginally Suitable Soils) class, $12.3 \%$ fits in $\mathrm{S} 2$ class (Moderately suitable) and $7.1 \%$ belongs to N1 class (Actually unsuitable and potentially suitable). Soil salinity, hydraulic conductivity and profile depth were considered the main restrictive factors for crop suitability in the study area.
\end{abstract}

Keywords: Spatial analyses, Soil mapping, GIS, Soil evaluation.

Increasing population pressure and changing human needs play a critical role in the competition for different uses for the same tract of land. Systematic land use planning is therefore needed to assure not only the improvement of the social conditions of the present but also the conservation of the environment for future generation. Moreover, land evaluation using a scientific process is important to assess the potential and constraints of a given land parcel for agricultural purposes (Rossiter, 1996). Recently, the cruel-effects of land use on the environment and environmental sustainability of agricultural production systems have become a subject of concern. The problems of declining soil fertility, overexploitation of natural resources and unrestricted soil erosion are associated with intensive agriculture in developing countries Lanen Van et al. (1992). Land evaluation analysis would resolve these issues while providing better land-use 
options to the farmers. Successful development soil evaluation program requires a comprehensive inventory of chemical and physical resources and environment. These are the basic elements of a land evaluation for regional land use planning. The aim of land evaluation is to provide land management with information, which will improve the quality of land use decisions.

Several systems for land evaluation in Egypt have been introduced. The system of Abdel Muttaleb and hussien (1985) was the first, who assured that environmental factors should be included in land evaluation. However, the involved calculation methods were tedious and could subject results for errors. Abd El-Sattar (1999) applied several evaluation systems for the soil of certain geomorphic units in Western Nile Delta and concluded that most of those systems have to be revised. Ismail et al. (2001) suggested The Applied System for Land Evaluation (ASLE) in arid and semi arid regions. They listed four major factors to define the land capability classification, which were: soil chemical and physical properties, environmental status, irrigation system and water qualities and soil fertility.

The main objective of this study is to assess the land spatial variability and evaluate new reclaimed soils in the study area of for irrigated agriculture.

The study area

El-Tina Plain, Egypt was chosen as study area. It is located in the northwestern part of Sinai Peninsula between longitudinal $32^{\circ} 22^{\prime \prime}$ and $32^{0}$ 29 "East and latitudinal $30^{\circ}$ 54" and $31^{\circ} 01$ " North (Fig.1), with total area of $127.29 \mathrm{Km}^{2}$.

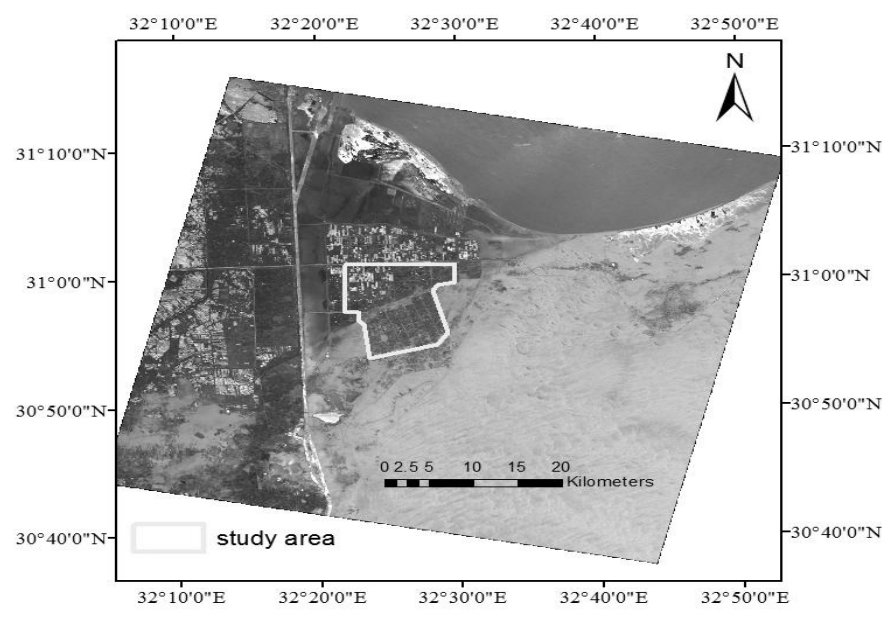

Fig. 1. Location of the study area.

Egypt. J. Soil Sci. 53, No. 1 (2013) 


\section{Geomorphology of the study area}

El-Taweel et al. (1997) reported that El- Tina plain is originally a part of the ancient belongs to the Nile Delta. Its elevation is at mean sea level or just above or below. The elevation varies between 0 and $2 \mathrm{~m}$ A.S.L. Elwan et al. (1983) and El-Taweel (1999) reported that the following mapping units were recognized map in North Western Sinai region.

1. Coastal sand beach (sand shore).

2. Individual sabkhas (El-Tina Plain).

3. Deflated sand terrain.

4. Level terrain with Aeolian sand deposits.

5. Mobile elevated sand dunes.

\section{Material and Methods}

Eighteen soil profiles were selected to represent the soils occurring in the studied area that include two main geomorphic units, i.e., El-Tina plain and level terrain with Aeolian sand deposits. Morphological description of selected soil profiles representing the different geomorphic units were carried out according to Soil Survey Staff (1993) and FAO (2006). Representative soil samples were collected from the subsequent soil layer of each profile for chemical and physical characterization of each sample.

\section{Laboratory analyses}

The collected soil samples were air dried ground and sieved through a $2 \mathrm{~mm}$ stainless steel sieve and stored in plastic vials for different analyses.

Soil color in both dry and moist conditions was measured with the aid of the Munsell Soil Color Charts (1975). Particle size distribution was carried out by hydrometer method Klute (1986). Hydraulic conductivity was estimated theoretically by using Rosetta Version 1.0 (Marcel, 1999). Furthermore, Chemical properties were carried out according to the standard method published by Page et al. (1982) and Baruah \& Barthakur (1997).

\section{Soil classification}

USDA Soil Taxonomy (1999) was used to classify different soil profiles. The soil correlation between the physiographic and taxonomy units were designed in order to identify the major soil sets of the different studied soils.

\section{Geographic Information Systems (GIS)}

ArcGIS 9.2 software was used to develop a GIS framework for the spatial analysis and data management. Soil data were then interpolated using the Inverse Distance Weighted (IDW), which based on the extent of similarity of cells, to generate one grid for each of soil attributes and produce the maps of them. 


\section{Land evaluation}

Land capability classification was done based on the land characteristics of the mapping units of the studied area using the tables of rating suggested by Sys and Verheye (1974) and Zakarya (2009).

Calculation of capability index (CI) was applied using Zakarya (2009) models according to the following:

Different soil factors affecting land evaluation are divided into:

- Physical factors (P) include soil texture (A), soil depth (B), slope (G) and hydraulic conductivity $(\mathrm{H})$.

- Chemical factors (C) include salinity (S), $\mathrm{CaCO}_{3}(\mathrm{C})$ and irrigation water quality $\left(\mathrm{W}_{\mathrm{i}}\right)$, then following equations were followed:

$\log (\mathrm{P})=\left[\log \left(\mathrm{A} * \mathrm{~B}^{*} \mathrm{~F}^{*} \mathrm{H}\right)\right] / 4$

$\log (\mathrm{C})=[\log (\mathrm{S} * \mathrm{C} * \mathrm{Wi})] / 3$.

$\mathrm{CI}=\mathrm{P} * \mathrm{C} / 100$.

\section{Results and Discussion}

Soils of south El-Tina plain

The soil of the study could be divided into two land types, namely soils of ElTina plain and level terrain with Aeolian sand deposits.

\section{Soils of El-Tina plain}

These soils are almost flat to very gently slopping and mainley adjacent to Mediterranean Sea from the north and Suez Canal from the west. The morphological description of some represented soil profiles are shwon in Table 1. The studied area includes three morphological sub-units, i.e., dry and wet sabkhas, sandy flat and wet sand flat. These soils are almost flat to very gently slopping and the elevation between $0.5-2 \mathrm{~m}$ A.S.L . The soils are characterized by extremely high content of salt and presence of salt crust, about $2 \mathrm{~cm}$ on the surface, while the water table is generally high and/or low. The parent materials of the soils consist of Fluvio Marine deposits or Lacustrine deposits and Aeolian sand deposits. soils of this unit are well represented by profiles No.(1 to 13$)$.

Results in Table 2 and Fig. $2 \& 3$ show that the texture of the studied soils are sandy clay loam, sandy loam, loam and sandy. Soil hydraulic conductivity values indicate that the infltration rate are very slow to moderate and ranged between 0.9 and $13.7 \mathrm{~mm} / \mathrm{hr}$. The organic matter content ranged between 0.2 and $2.7 \%$. This is may be due to common humified and fresh residuals of organic residues (Fish Ponds) and the high salt content which slow down the activity of microorganisms for decomposing the organic matter (El-Khattib, 1978). These results are also in agreement with the data of Noha (2000). Electrical conductivity values of the soil saturation extract indicated that the soils are extremely saline to moderately saline and ranged between 6 and $96 \mathrm{dS} / \mathrm{m}$, while the soil reaction is slightly alkaline to alkaline and $\mathrm{pH}$ ranged from 7.90 to 8.68 . The soils have relatively high calcium carbonate content in some of the studied soil samples as in profile Nos. (6,7 and 12). The high Egypt. J. Soil Sci. 53, No. 1 (2013) 
content of $\mathrm{CaCO}_{3}$ could be rendered to the existence of common to medium shell fragments, while low content of $\mathrm{CaCO}_{3}$ was achieved in all soil profiles.

TABLE 1. Morphological description of selected soil profiles representing the studied soil units.

\begin{tabular}{|c|c|c|c|}
\hline $\begin{array}{l}\text { Land } \\
\text { form }\end{array}$ & $\begin{array}{l}\text { Profile } \\
\text { No }\end{array}$ & \begin{tabular}{|l|}
$\begin{array}{l}\text { Soil } \\
\text { depth } \\
(\mathbf{c m})\end{array}$ \\
\end{tabular} & Profile Description \\
\hline \multirow{9}{*}{ 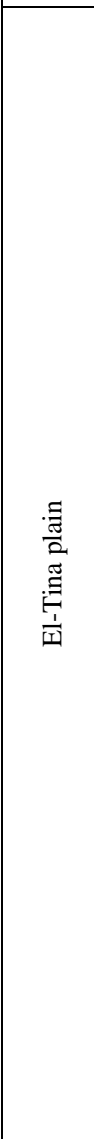 } & \multirow{3}{*}{1} & $0-15$ & $\begin{array}{l}\text { Dark gray }(5 \mathrm{Y}, 4 / 1) \text { dry; Very dark gray }(5 \mathrm{Y}, 3 / 1) \text { moist; silty Clay loam; } \\
\text { Strong medium subangular blocky structure; Firm; Sticky; plastic; } \\
\text { Moderately effervescence with HCl; Few spots of organic residues; Very } \\
\text { few very fine soft gypsum; Few very fine pores; Abrupt smooth boundary. }\end{array}$ \\
\hline & & $15-30$ & $\begin{array}{l}\text { Light olive gray }(5 \mathrm{Y}, 6 / 2) \text { dry; Olive gray }(5 \mathrm{Y}, 4 / 2) \text { moist; Silty loam; } \\
\text { Weak fine subangular blocky structure; Firm; sticky; plastic; Few spots of } \\
\text { organic resides; Few fine soft gypsum; Moderately effervescence with } \\
\mathrm{HCl} \text {; Clear smooth boundary. }\end{array}$ \\
\hline & & $30-60$ & $\begin{array}{l}\text { Light olive gray }(5 \mathrm{Y}, 6 / 2) \text { dry; Olive gray }(5 \mathrm{Y}, 4 / 2) \text { moist; Silty loam; } \\
\text { Weak fine subangular blocky structure; sticky; plastic; Moderately } \\
\text { effervescence with } \mathrm{HCl} \text {; Few spots of organic residues; Few very fine to } \\
\text { medium pores. }\end{array}$ \\
\hline & \multirow{3}{*}{5} & $0-15$ & $\begin{array}{l}\text { Light brown gray (10YR, 6/2) dry; Very dark grayish brown }(10 \mathrm{YR}, 3 / 2) \\
\text { moist; Sandy loam; Massive; Firm; sticky; plastic; Very Slight } \\
\text { effervescence with } \mathrm{HCl} \text {; Common well developed gypsum crystals; Very } \\
\text { few very fine pores; Clear smooth boundary. }\end{array}$ \\
\hline & & $15-30$ & $\begin{array}{l}\text { Grayish brown (10YR, 5/2) dry; Very dark grayish brown }(10 \mathrm{YR}, 3 / 2) \\
\text { moist; Sandy loam; Massive; Firm; sticky; plastic; Slight effervescence } \\
\text { with } \mathrm{HCl} \text {; Few fine soft gypsum crystals; Few very fine pores; Diffuse } \\
\text { smooth boundary. }\end{array}$ \\
\hline & & $30-80$ & $\begin{array}{l}\text { Gray (10YR, 5/1) dry; Very dark grayish brown (10YR, 3/2) moist; Loam; } \\
\text { Massive; Firm; Very sticky; Very plastic; Slight effervescence with } \mathrm{HCl} \text {; } \\
\text { Common well developed gypsum crystals; Very few very fine pores. }\end{array}$ \\
\hline & \multirow{3}{*}{12} & $0-15$ & $\begin{array}{l}\text { Brown }(10 \mathrm{YR}, 5 / 3) \text { dry ; Dark brown }(10 \mathrm{YR}, 3 / 3) \text { moist ; loam ; Massive ; } \\
\text { Firm ; Sticky ; Plastic ; moderately effervescence with } \mathrm{HCl} \text {; Few spots of } \\
\text { organic residues; Common well developed gypsum crystals ; Clear smooth } \\
\text { boundary. }\end{array}$ \\
\hline & & $15-30$ & $\begin{array}{l}\text { Olive gray }(5 \mathrm{Y}, 5 / 2) \text { dry; Olive gray }(5 \mathrm{Y}, 4 / 2) \text { moist; Loam; Weak fine } \\
\text { subangular blocky structure; Hard; Very sticky; Very plastic; moderately } \\
\text { visible effervescence with } \mathrm{HCl} \text {; few fine soft shell fragments; Few spots of } \\
\text { organic residues; Few fine pores; Clear smooth boundary. }\end{array}$ \\
\hline & & $30-80$ & $\begin{array}{l}\text { Light brownish gray }(2.5 \mathrm{Y}, 6 / 2) \text { dry; Dark grayish brown (2.5 Y 4/2) } \\
\text { moist; Sandy loam; Massive; Firm; sticky; plastic; Strong visible } \\
\text { effervescence with HCl; many fine soft shell fragments; Few spots of } \\
\text { organic residues; Very few fine pores; Few fine soft gypsum }\end{array}$ \\
\hline \multirow{3}{*}{ 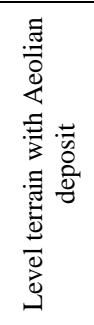 } & \multirow{3}{*}{14} & $0-15$ & $\begin{array}{l}\text { Very pale brown }(10 \mathrm{YR}, 7 / 3) \text { dry ; Grayish brown }(10 \mathrm{YR}, 5 / 2) \text { moist ; } \\
\text { Sand ; Single grain ; Loose ; Non-Sticky ; Non-Plastic ; Slight } \\
\text { effervescence with } \mathrm{HCl} \text {; Few fine roots ; Few fine pores ; Clear smooth } \\
\text { boundary. }\end{array}$ \\
\hline & & $15-30$ & $\begin{array}{l}\text { Light yellowish brown (10YR,6/4) dry ; yellowish brown (10YR,5/4) } \\
\text { moist ; Sand ; Loose ; Non-sticky ; Non-plastic ; Slight effervescence with } \\
\mathrm{HCl} \text {; Very few fine pores ; Few fine roots ; Clear smooth boundary. }\end{array}$ \\
\hline & & $30-120$ & $\begin{array}{l}\text { Light yellowish brown (10YR,6/4) dry ; yellowish brown (10YR,5/4) } \\
\text { moist ; Sand ; Massive ; friable ; Non-sticky ; Non-plastic ; Slight } \\
\text { effervescence with HCl. }\end{array}$ \\
\hline
\end{tabular}


TABLE 2. Some physical and chemical proprieties of the study area profiles.

\begin{tabular}{|c|c|c|c|c|c|c|c|c|c|c|c|c|}
\hline 胥 & $\begin{array}{l}\stackrel{2}{z} \\
\dot{\vec{g}}\end{array}$ & 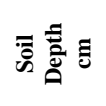 & 졸 & के & 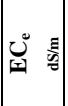 & $\sum_{0}^{\circ}$ & $\overbrace{\tilde{J}}^{0}:$ & 矛。 & 䋅。 & $\vec{\Xi} 0^{\circ}$ & 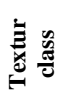 & $\stackrel{2}{m}^{m}$ \\
\hline \multirow{40}{*}{ 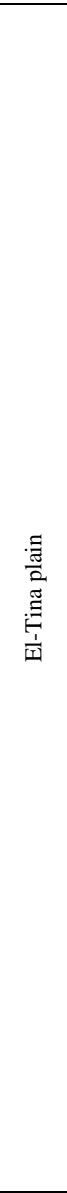 } & \multirow{3}{*}{1} & $0-15$ & 7.95 & 150 & 25 & 1.11 & 4.4 & 0.00 & 73 & 27 & SiCL & 1.8 \\
\hline & & $15-30$ & 8.23 & \begin{tabular}{|l|}
170 \\
\end{tabular} & 28 & 1.05 & 3.7 & 20.00 & 67 & 16 & SiL & 1.8 \\
\hline & & $30-60$ & 8.27 & 163 & 48 & 1.01 & 3.1 & 24.00 & 56 & 20 & SiL & 2 \\
\hline & \multirow{4}{*}{2} & $0-15$ & 8.39 & 95 & 51 & 0.58 & 1.5 & 40.00 & 47 & 13 & $\mathrm{~L}$ & 1.8 \\
\hline & & $15-30$ & 8.36 & 100 & 43 & 0.5 & 1.0 & 40.00 & 47 & 13 & $\mathrm{~L}$ & 1.8 \\
\hline & & $30-60$ & 8.27 & 110 & 52 & 0.7 & 0.7 & 40.00 & 52 & 8 & $\mathrm{SiL}$ & 2 \\
\hline & & $60-80$ & 8.20 & 96 & 96 & 0.65 & 0.6 & 43.00 & 47 & 10 & $\mathrm{~L}$ & 1.8 \\
\hline & \multirow{3}{*}{3} & $0-15$ & 8.35 & 80 & 34 & 0.38 & 0.6 & 43.00 & 49 & 8 & $\mathrm{~L}$ & 1.7 \\
\hline & & $15-30$ & 8.48 & 90 & 20 & 0.5 & 0.8 & 47.00 & 46 & 7 & $\mathrm{~L}$ & 2.3 \\
\hline & & $30-70$ & 8.60 & 135 & 22 & 0.42 & 0.9 & 43.00 & 50 & 7 & $\mathrm{SiL}$ & 2.2 \\
\hline & \multirow{3}{*}{4} & $0-15$ & 8.45 & 80 & 43 & 0.45 & 0.9 & 60.00 & 36.7 & 3.3 & $\mathrm{SL}$ & 1.5 \\
\hline & & $15-30$ & 8.45 & 95 & 31 & 0.58 & 0.5 & 63.30 & 31.6 & 5 & SL & 1.6 \\
\hline & & $30-70$ & 8.44 & 85 & 30 & 0.55 & 0.8 & 60.00 & 35 & 5 & SL & 1.9 \\
\hline & \multirow{3}{*}{5} & $0-15$ & 8.31 & 65 & 47 & 0.47 & 1.1 & 73.30 & 21.6 & 5 & SL & 1.5 \\
\hline & & $15-30$ & 8.51 & 90 & 30 & 0.58 & 1.1 & 63.30 & 30 & 6.6 & SL & 1.8 \\
\hline & & $30-80$ & 8.44 & 100 & 31 & 0.58 & 1.0 & 43.30 & 41.6 & 15 & $\mathrm{~L}$ & 1.8 \\
\hline & \multirow{3}{*}{6} & $0-15$ & 8.32 & 80 & 15 & 0.58 & 10.9 & 55.00 & 36.6 & \begin{tabular}{|l|}
8.3 \\
\end{tabular} & SL & 1.77 \\
\hline & & $15-30$ & 8.40 & 75 & 17 & 0.51 & 8.5 & 61.60 & 29.9 & 8.5 & SL & 1.81 \\
\hline & & $30-60$ & 8.62 & 150 & 20 & 0.51 & 9.5 & 46.70 & \begin{tabular}{|l|}
43.3 \\
\end{tabular} & 10 & $\mathrm{~L}$ & 1.78 \\
\hline & \multirow{3}{*}{7} & $0-15$ & 8.68 & 50 & 14 & 0.51 & 3.3 & 71.60 & 18.3 & 10 & SL & 1.86 \\
\hline & & $15-30$ & 8.50 & 70 & 8 & 0.51 & 4.7 & 53.30 & 36.6 & 10 & SL & 1.69 \\
\hline & & $30-100$ & 8.40 & 80 & 6 & 0.58 & 7.6 & 56.70 & 31.6 & 11.7 & SL & 1.67 \\
\hline & \multirow{3}{*}{8} & $0-15$ & 8.25 & 65 & 22 & 0.58 & 2.4 & 46.60 & 43.3 & 10 & $\mathrm{~L}$ & 1.59 \\
\hline & & $15-30$ & 8.20 & 90 & 34 & 0.58 & 0.1 & 36.60 & 52.3 & 11 & $\mathrm{SiL}$ & 1.89 \\
\hline & & $30-100$ & 8.20 & 75 & 26 & 0.42 & 0.3 & 33.30 & 53.2 & 13.5 & $\mathrm{SiL}$ & 1.92 \\
\hline & \multirow{3}{*}{9} & $0-15$ & 8.10 & 66 & 22 & 0.4 & 4.8 & 53.30 & 38.2 & \begin{tabular}{|l|}
8.5 \\
\end{tabular} & SL & 1.7 \\
\hline & & $15-30$ & 8.30 & 56 & 33 & 0.4 & 1.6 & 55.00 & \begin{tabular}{|l|}
36 \\
\end{tabular} & 9 & SL & 1.7 \\
\hline & & $30-70$ & 8.20 & 60 & 39 & 0.3 & 5.6 & 47.00 & 40 & 13 & $\mathrm{~L}$ & 1.9 \\
\hline & \multirow{3}{*}{10} & $0-15$ & 8.40 & 50 & 19 & 2.2 & 3 & 50.00 & 38 & 12 & $\mathrm{~L}$ & 1.5 \\
\hline & & $15-30$ & 8.40 & 60 & 15 & 0.3 & 2.1 & 46.00 & 41 & 13 & $\mathrm{~L}$ & 1.8 \\
\hline & & $30-90$ & 8.20 & 50 & 28 & 0.3 & 4.2 & 50.00 & 35 & 15 & $\mathrm{~L}$ & 1.6 \\
\hline & \multirow{3}{*}{11} & $0-15$ & 7.90 & 80 & 17 & 0.6 & 4.2 & 23.00 & 64 & 13 & $\mathrm{SiL}$ & 1.8 \\
\hline & & $15-30$ & 8.30 & 93 & 21 & 0.7 & 0.7 & 23.30 & 61.6 & 15 & $\mathrm{SiL}$ & 1.8 \\
\hline & & $30-60$ & 8.30 & 102 & 38 & 0.6 & 1.9 & 40.00 & 46 & 14 & $\mathrm{~L}$ & 1.7 \\
\hline & \multirow{3}{*}{12} & $0-15$ & 8.30 & 81 & 10 & 2.7 & 3.4 & 40.00 & 48.3 & \begin{tabular}{|l|}
11.7 \\
\end{tabular} & $\mathrm{~L}$ & 1.8 \\
\hline & & $15-30$ & 8.10 & 74 & 8 & 0.5 & 7.8 & 41.70 & 45 & 13.3 & $\mathrm{~L}$ & 1.4 \\
\hline & & $30-80$ & 8.40 & 67 & 9 & 0.2 & 33.7 & 53.30 & 33.2 & 13.5 & SL & 1.6 \\
\hline & & $0-15$ & 8.40 & 56 & 21 & 0.4 & 2.3 & 63.30 & 29.3 & \begin{tabular}{|l|}
7.4 \\
\end{tabular} & SL & 1.5 \\
\hline & 13 & $15-30$ & 8.30 & 100 & 29 & 0.4 & 2.6 & 60.00 & 30 & 10 & SL & 1.7 \\
\hline & & $30-60$ & 8.30 & 69 & 30 & 0.4 & 1.9 & 63.30 & 29.3 & \begin{tabular}{|l|}
7.4 \\
\end{tabular} & SL & 1.6 \\
\hline \multirow{15}{*}{ 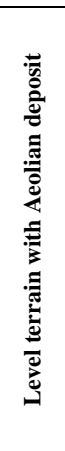 } & \multirow{3}{*}{14} & $0-15$ & 8.17 & 25 & 0.9 & 0.4 & 1.1 & 95 & 1.6 & 3.4 & $\mathrm{~S}$ & 1.4 \\
\hline & & $15-30$ & 8.52 & 21 & 1.0 & 0.3 & 0.8 & 96 & 2.2 & 1.8 & S & 1.5 \\
\hline & & $30-120$ & 8.47 & 25 & 0.9 & 0.2 & 0.5 & 96 & 2.0 & 2.0 & $\mathrm{~S}$ & 1.5 \\
\hline & & $0-15$ & 8.10 & 25 & 1.7 & 0.4 & 0.9 & 92 & 3.5 & 4.5 & S & 1.4 \\
\hline & 15 & $15-30$ & 8.19 & 23 & 2.3 & 0.4 & 0.5 & 95 & 2.3 & 2.7 & $\mathrm{~S}$ & 1.5 \\
\hline & & $30-120$ & 7.86 & 23 & 2.2 & 0.2 & 0.6 & 96 & 1.5 & 2.5 & $\mathrm{~S}$ & 1.5 \\
\hline & 16 & $0-15$ & 8.17 & 23 & 0.9 & 0.4 & 1.1 & 93 & 3.2 & 3.8 & $S$ & 1.4 \\
\hline & & $15-30$ & 8.52 & 21 & 1.1 & 0.3 & 1.2 & 95 & 2.2 & 2.8 & S & 1.5 \\
\hline & & $30-120$ & 8.47 & 22 & 0.9 & 0.3 & 0.9 & 96 & 2.0 & 2.0 & $\mathrm{~S}$ & 1.5 \\
\hline & 17 & $0-15$ & 8.10 & 23 & 2.7 & 0.5 & 0.5 & 94 & 3.6 & 2.4 & $\mathrm{~S}$ & 1.4 \\
\hline & & $15-30$ & 8.19 & 21 & 2.3 & 0.3 & 0.5 & 95 & 3.0 & 2.0 & $S$ & 1.5 \\
\hline & & $30-120$ & 7.86 & 21 & 3.2 & 0.2 & 0.4 & 95 & 3.0 & 2.0 & S & 1.5 \\
\hline & 18 & $0-15$ & 8.17 & 22 & 1.0 & 0.3 & 0.6 & 95 & 2.0 & 3.0 & S & 1.4 \\
\hline & & $15-30$ & 8.52 & 21 & 1.2 & 0.2 & 0.5 & 96 & 1.8 & 2.2 & $\mathrm{~S}$ & 1.5 \\
\hline & & $30-120$ & 8.47 & 22 & 0.9 & 0.2 & 0.5 & 96 & 2.0 & 2.0 & S & 1.5 \\
\hline
\end{tabular}

Egypt. J. Soil Sci. 53, No. 1 (2013) 

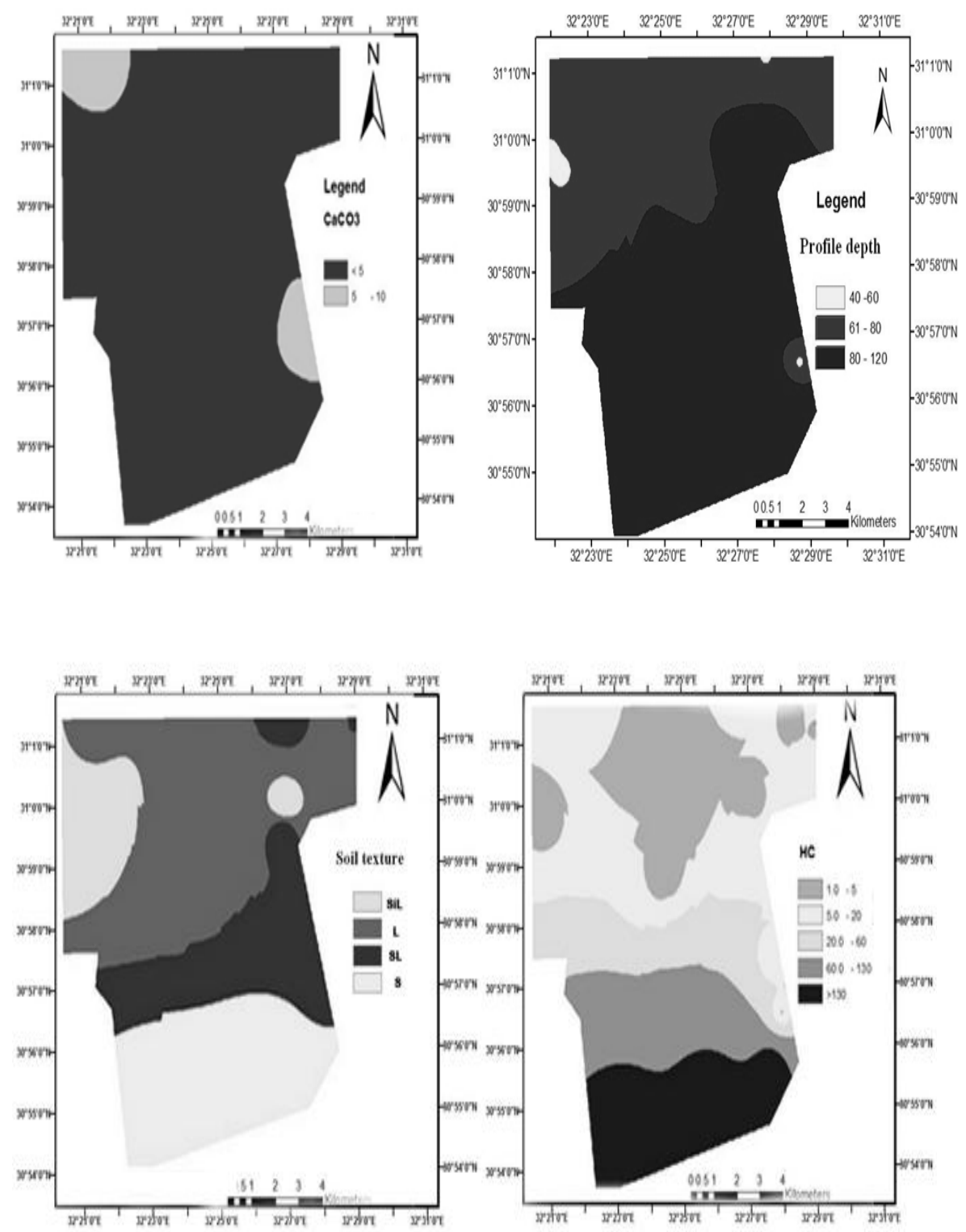

Fig. 2. Spatial distribution of $\mathrm{CaCO}_{3}$ content, profile depth, soil texture and hydraulic conductivity in the study area. 


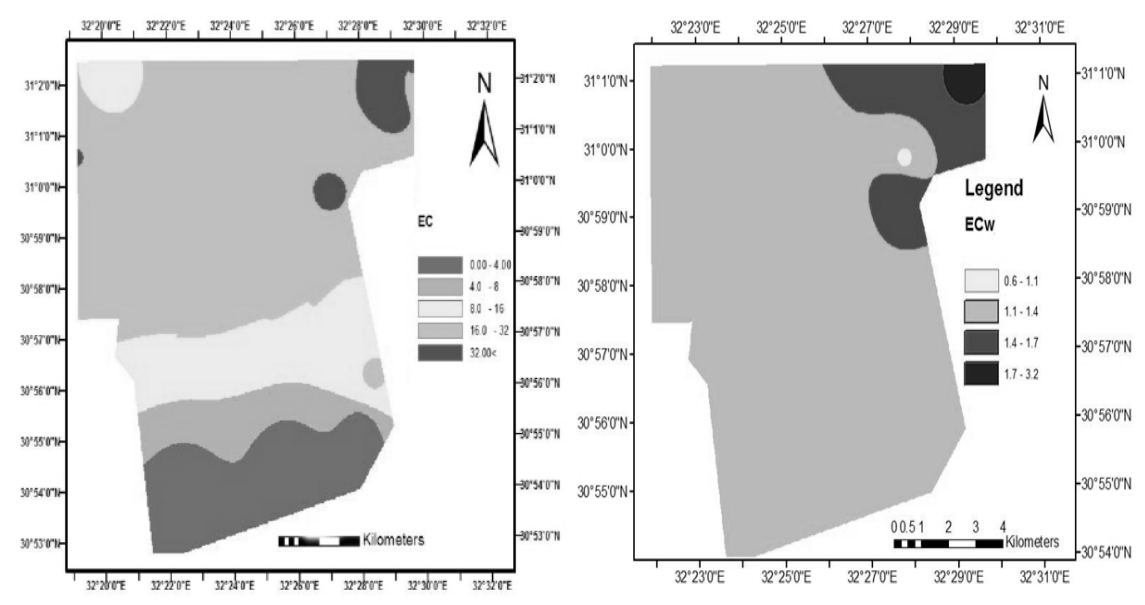

Fig. 3. Spatial distribution of $\mathrm{EC}_{\mathrm{e}}$ and $\mathrm{EC}_{\mathrm{iw}}$ in the study area.

The data of soluble cations and anions showed that, sodium is the dominant cation in the different layers in all the studied soil profiles, followed by $\mathrm{Mg}^{++}$, $\mathrm{Ca}^{++}$and $\mathrm{K}^{+}$ions, while the anionic composition is dominated by $\mathrm{Cl}^{-}$ion followed by $\mathrm{SO}_{4}{ }^{=}$and $\mathrm{HCO}_{3}{ }^{-}$.

\section{Soils of the level terrain with aeolian sand deposits}

These soils are almost flat to gently undulating. The surface of these soils are mostly cover by sand ripples. Soils of this unit are well represented by profile No.(14, 15, 16, 17 and 18). The data show that soil texture is sandy. Soil hydraulic conductivity values indicate that the infltration rate are very fast more than $130 \mathrm{~mm} / \mathrm{hr}$. Organic matter content is very low, ranging between 0.20 to $0.40 \%$. EC values are ranging between 0.9 and $3.2 \mathrm{dS} / \mathrm{m}$. The soil reaction is slightly alkaline to alkaline and $\mathrm{pH}$ ranges from 7.8 to 8.5 . The chemical composition of the soil saturation extract indicates that the dominancy of $\mathrm{Na}^{+}$ followed by $\mathrm{Ca}^{++}, \mathrm{Mg}^{++}$and $\mathrm{K}^{+}$. Chloride $(\mathrm{Cl})$ and/or sulphates $\left(\mathrm{SO}_{4}{ }^{=}\right)$are the dominant anions followed by $\mathrm{HCO}_{3}{ }^{-}$.

\section{Soil classification}

Based on the morphological description and data analyses, the soils of the studied area were classified into two orders as Aridisols (Typic Aquisalids) and Entisols (Typic Torriorthents, Typic Torripsamments). 


\section{Land evaluation}

Capability indices of different classes were calculated according to values of Sys and Verheye (1974) and Zakarya (2009). The soils of the studied area were placed in the capability classes S2, S3 and N1 (Fig. 4). Rating values of different land parameters, capability index $(\mathrm{CI})$ and limitation factors are presented in Table 3. The differences between those classes are explained in the following:

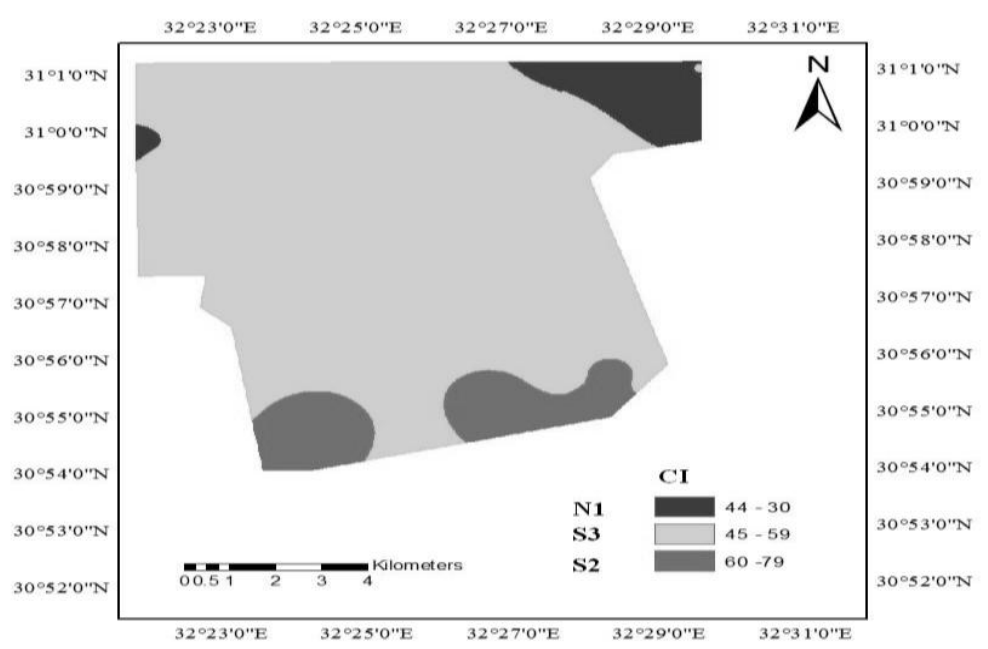

Fig. 4. Land capability classes of the studied area.

\section{Class S2 (Moderately suitable)}

Suitable soils including profile no $(7,12,14,15,16,17$ and 18) representing $15.66 \mathrm{Km}^{2}(12.7 \%)$ of the total area. The data show that the CI values range from 60 to 60.3 . These soils are characterized by presence of available water for irrigation from El-Salam canal, with $\mathrm{EC}_{\mathrm{iw}}$ varied from $(0.95-1.1 \mathrm{dS} / \mathrm{m})$. The soils have moderate to deep profile, moderate to well drained, slope less than $1 \%$, hydraulic conductivity moderate to very vast $(5.58-162 \mathrm{~mm} / \mathrm{hr})$, their soil salinity varied from $(0.96-14.28 \mathrm{dS} / \mathrm{m})$ and the $\mathrm{CaCO}_{3}$ less than $10 \%$. The main limitation factors are texture, hydraulic conductivity and salinity, therefore these soils need to careful management to decrease salinity and alkalinity and improvement of the drainage conditions. Moderately salinity in these soils irrigated by good quality water (Wi $>80 \%$ ) is not a problem and can be corrected very easily. The main problem of sandy texture soils is that related to their fertility and plant nutrient level and this is not a limitation nowadays under the new fertigation practices. These soils need low technical advisory services. 
TABLE 3. Rating of limitation and current Capability classes of the studied area.

\begin{tabular}{|c|c|c|c|c|c|c|c|c|c|c|c|c|}
\hline \multirow{2}{*}{$\begin{array}{l}\text { Prof. } \\
\text { No. }\end{array}$} & \multicolumn{4}{|c|}{ Physical index(P) } & \multicolumn{3}{|c|}{ Chemical index (C) } & \multirow[b]{2}{*}{$\mathbf{P}$} & \multirow[b]{2}{*}{$\mathbf{C}$} & \multirow[b]{2}{*}{ CI } & \multirow[b]{2}{*}{ Class } & \multirow{2}{*}{$\underset{* *}{\text { Limitation }^{-}}$} \\
\hline & $\begin{array}{c}\text { Texture } \\
\text { (A) }\end{array}$ & $\begin{array}{c}\text { Depth } \\
\text { (B) }\end{array}$ & $\begin{array}{l}\text { Slope } \\
\text { (G) }\end{array}$ & $\begin{array}{l}\mathrm{HC} \\
\text { (H) }\end{array}$ & $\begin{array}{c}\text { Salinity } \\
\text { (S) }\end{array}$ & $\begin{array}{c}\mathrm{CaCO3} \\
\text { (C) }\end{array}$ & $\begin{array}{l}\mathbf{E C}_{\text {iw }} \\
(\mathrm{Wi})\end{array}$ & & & & & \\
\hline 1 & 100 & 80 & 100 & 50 & 20 & 100 & 80 & 79.53 & 54.29 & 43.2 & N1 & $\mathrm{H}, \mathrm{S}$ \\
\hline 2 & 100 & 90 & 100 & 50 & 20 & 100 & 50 & 81.90 & 46.41 & 38.0 & N1 & $\mathrm{H}, \mathrm{S}$ \\
\hline 3 & 100 & 90 & 100 & 25 & 20 & 100 & 65 & 68.87 & 50.66 & 34.9 & N1 & $\mathrm{H}, \mathrm{S}, \mathrm{Wi}$ \\
\hline 4 & 90 & 90 & 100 & 100 & 20 & 100 & 65 & 94.86 & 50.66 & 48.1 & S3 & $\mathrm{S}, \mathrm{Wi}$ \\
\hline 5 & 90 & 90 & 100 & 85 & 20 & 100 & 65 & 91.10 & 50.66 & 46.1 & S3 & S,Wi \\
\hline 6 & 90 & 80 & 100 & 75 & 20 & 80 & 95 & 85.72 & 53.37 & 45.7 & S3 & $\mathrm{S}$ \\
\hline 7 & 90 & 100 & 100 & 85 & 40 & 80 & 80 & 93.52 & 63.50 & 60 & $\mathrm{~S} 2$ & $S$ \\
\hline 8 & 100 & 100 & 100 & 50 & 20 & 100 & 95 & 84.09 & 57.59 & 48.3 & S3 & $\mathrm{H}, \mathrm{S}$ \\
\hline 9 & 90 & 90 & 100 & 50 & 20 & 100 & 95 & 79.77 & 57.59 & 45.9 & S3 & $\mathrm{H}, \mathrm{S}$ \\
\hline 10 & 100 & 100 & 100 & 50 & 20 & 100 & 80 & 84.09 & 54.29 & 45.7 & S3 & $\mathrm{H}, \mathrm{S}$ \\
\hline 11 & 100 & 80 & 100 & 50 & 20 & 100 & 95 & 79.53 & 57.49 & 45.7 & S3 & $\mathrm{H}, \mathrm{S}$ \\
\hline 12 & 100 & 90 & 100 & 85 & 40 & 80 & 80 & 93.52 & 63.50 & 60 & S2 & $S$ \\
\hline 13 & 90 & 80 & 100 & 85 & 20 & 100 & 65 & 88.45 & 50.66 & 44.8 & N1 & $\mathrm{S}, \mathrm{Wi}$ \\
\hline 14 & 75 & 100 & 95 & 25 & 100 & 100 & 80 & 64.97 & 92.83 & 60.3 & S2 & $\mathrm{A}, \mathrm{H}$ \\
\hline 15 & 75 & 100 & 95 & 25 & 100 & 100 & 80 & 64.97 & 92.83 & 60.3 & S2 & $\mathrm{A}, \mathrm{H}$ \\
\hline 16 & 75 & 100 & 95 & 25 & 100 & 100 & 80 & 64.97 & 92.83 & 60.3 & S2 & $\mathrm{A}, \mathrm{H}$ \\
\hline 17 & 75 & 100 & 95 & 25 & 100 & 100 & 80 & 64.97 & 92.83 & 60.3 & S2 & $\mathrm{A}, \mathrm{H}$ \\
\hline 18 & 75 & 100 & 95 & 25 & 100 & 100 & 80 & 64.97 & 92.83 & 60.3 & S2 & $\mathrm{A}, \mathrm{H}$ \\
\hline
\end{tabular}

*CI - Capability index

**Soil texture (A), soil depth (B), slope (G), hydraulic conductivity (H), Salinity (S), $\mathrm{CaCO}_{3}(\mathrm{C})$ and irrigation water quality $\left(\mathrm{W}_{\mathrm{i}}\right)$.

\section{Class S3 (Marginally suitable soils)}

Marginally suitable soils including profile no (4, 5, 6, 8, 9, 10 and 11) representing $102.6 \mathrm{~km}^{2}(80.6 \%)$ of the total area. The data show that the CI values range from 48.1 to 45.7 . These soils are characterized by presence of available water for irrigation from El-Salam canal with $\mathrm{EC}_{\mathrm{iw}}$ varied from (1.03 $1.7 \mathrm{dS} / \mathrm{m})$. The soils have moderately deep profile, poor to moderate drained, slope less than $1 \%$, hydraulic conductivity slow to moderate $(1.19-13.75$ $\mathrm{mm} / \mathrm{hr})$, their soil salinity varied from $(15.45-47 \mathrm{dS} / \mathrm{m})$. The main limitation factors are hydraulic conductivity and salinity, therefore these soils need to salt leaching and drainage improvement. These soils need moderate technical services. 
Class N1 (Actually unsuitable and potentially suitable soils)

The obtained data reveal clearly that soils having capability class N1 have three severe limitations, salinity, hydraulic conductivity and salinity of irrigation water, therefore these soils need to removing salt crust, leaching salt, added some amendment such as gypsum and drainage improvement are much recommended for improving the land capability.

Actually unsuitable and potentially suitable Soils including profile no (1, 2, 3 and 13) representing $9.03 \mathrm{~km}^{2}(7.1 \%)$ of the total area. These soils need very high technical services of specialists. Conservation and management processes must be applied more frequently and intensively.

\section{References}

Abd El-Muttelib, M.A. and Hussein S.A. (1985) "Evaluation Soils Under Cultivation", Hand Book (Arabic addition).

Abd El-Sattar, M.M. (1999) Study on Soils of Certain Geomorphologic units, west of the Nile Delta, and their land suitability Ph. D. Thesis, Fac. of Agric., Cairo Univ., Egypt.

Baruah, T.C. and Barthakur, H.P. (1997) "A Text Book of Soil Analysis", pp. 4, Vikas Publishing House PVT LTD, New Delhi.

El-Khattib, H.M. (1978) Studies on diagnostic features of the Egyptian salt-affected soils. M. Sc. Thesis, Fac. of Agric., Cairo Univ., Egypt.

El-Taweel, M., Abdel Rahman, S. and Abdel Rahman, M. (1997) Assessment and evaluation of salt affected soils in El Tina Plain, Sinai Peninsula,Egypt.Egypt. J.Soil Sci.37:141-152.

El-Taweel, M.I. (1999) Soil map of Northern Sinai Governorate using remote sensing techniques. J. Agric. Sci. Mans. Univ. 24: 4307-4316.

Elwan, A.A. , Harga, A.A., Elkadi, H. and Eldemerdash, S. (1983) Preliminary studies on the soils of North Sinai Peninsula based on Aerial Photo- Interpretation. Egypt. J. Soil Sci. 23: 37-42.

F.A.O. (2006) "Guidelines for Soil Profile Description", $4^{\text {th }}$ ed., Food and Agriculture Organization of the United Nations, Rome.

Ismail, H.A., Morsy, I., El-Zahaby, E.M. and El-Nagar, F.S. (2001) A developed expert system for land use planning by coupling land information system and modeling. Alex. J. Agric. Res. 46 (3), 141.

Klute, A. (1986) "Methods of Soil Analysis Part 1. Physical and Mineralogical Methods", $2^{\text {nd }}$ ed., Soil Science Society of America, Inc. Madison, Wisconsin, USA.

Lanen Van, H. A. J., Diepen Van, C. A., Reinds, G. J. and Koning, G. H. J. (1992) Physical land valuation methods and GIS to explore the crop growth potential and its effects within the European communities. Agric. Syst. 38: 307-328. 
Marcel, S. (1999) Predicting soil hydraulic parameters from basic soil data. Rosetta Version 1.0

Munsell Soil Color Charts (1975) Soil Survey Manual. Baltimore, Mary Land.

Noha, H., SH. (2000) Land use mapping for selected areas of El-Salam Canal commandSinai, using remote sensing and GIS. M.Sc. Thesis, Fac. of Agric., Cairo Univ., Egypt.

Page, A.L., Miller, R.H. and Keeney, D.R. (1982) "Chemical and Microbiological Properties", Part 2, $2^{\text {nd }}$ ed., pp. 13-26 and 149-223, Agronomy 9, American Society of Agronomy.

Rossiter, G. D. (1996) A theoretical frame work for land evaluation. Goederma 72: 165-190.

Soil Survey Division Staff. (1993) "Soil Survey Manual", Soil Conservation Service U.S., Department of Agriculture Handbook 18.

Soil Taxonomy (1999) "A Basic System of Soil Classification for Making and Interpreting Soil Surveys", $2^{\text {nd }}$ ed., by soil survey staff, USDA, Agriculture handbook number 436 .

Sys, C. and Verheye, W. (1974) Land evaluation for irrigation of arid regions by the use of the parametric method. Trans. Loth Intern. Soil Congr., Mosscow 10:149-155.

Zakarya, Y.M. (2009) Land Resources Assessment Using GIS, Expert Knowledge and Remote Sensing in the Desert Environment., Ph.D.Thesis, Fac. Agri., Ain Shams Univ., Egypt. 


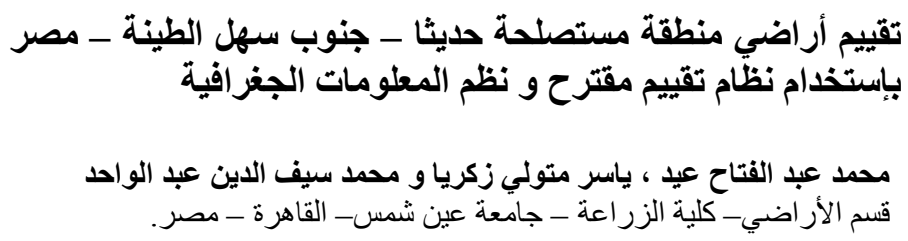

يهذف هذا البحث إلي تقييم مساحة 127.29 كم² للزراعة المروية من الأراضي

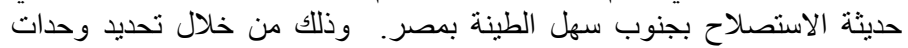

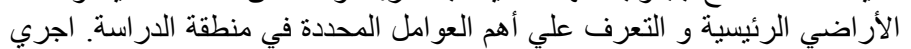

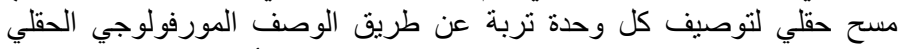

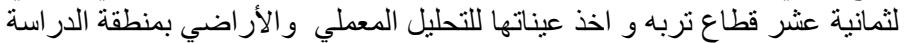

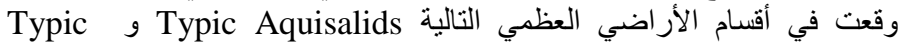

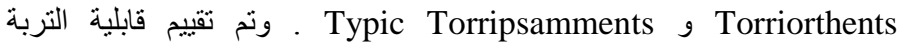
للاستزر اع باستخدام نموذج تقييم معدل محليا بالتكامل مع نظم المعلومات الجغر الفئ الفية

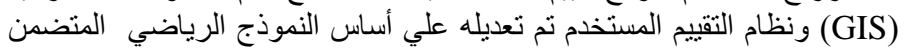

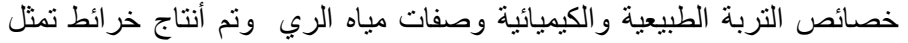

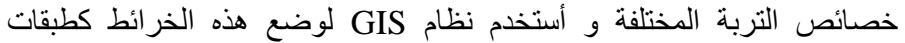

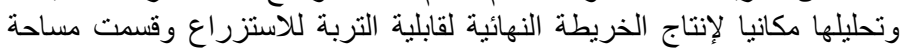

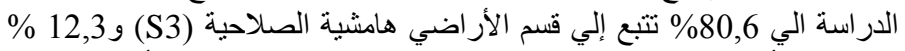

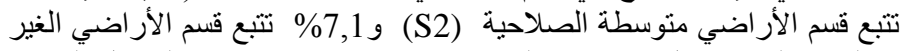

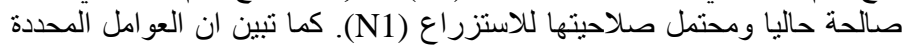

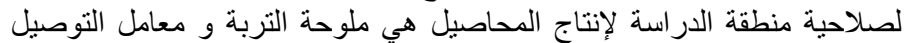
الهيدروليكي للتربة و عمق قطاع التربة. 